Supplementary Information

\title{
Calibration-free measurement of phenylalanine levels in blood using an electrochemical aptamer- based sensor suitable for point-of-care applications
}

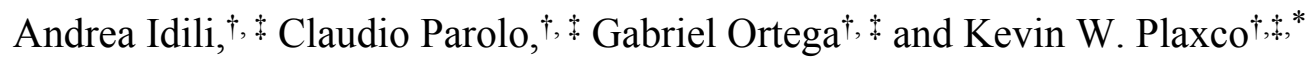

Department of Chemistry and Biochemistry and ${ }^{+C e n t e r}$ for Bioengineering, University of

California, Santa Barbara; Santa Barbara, CA 93106, United States

*Corresponding Author, E-mail: kwp@ucsb.edu 
SUPPORTING FIGURES

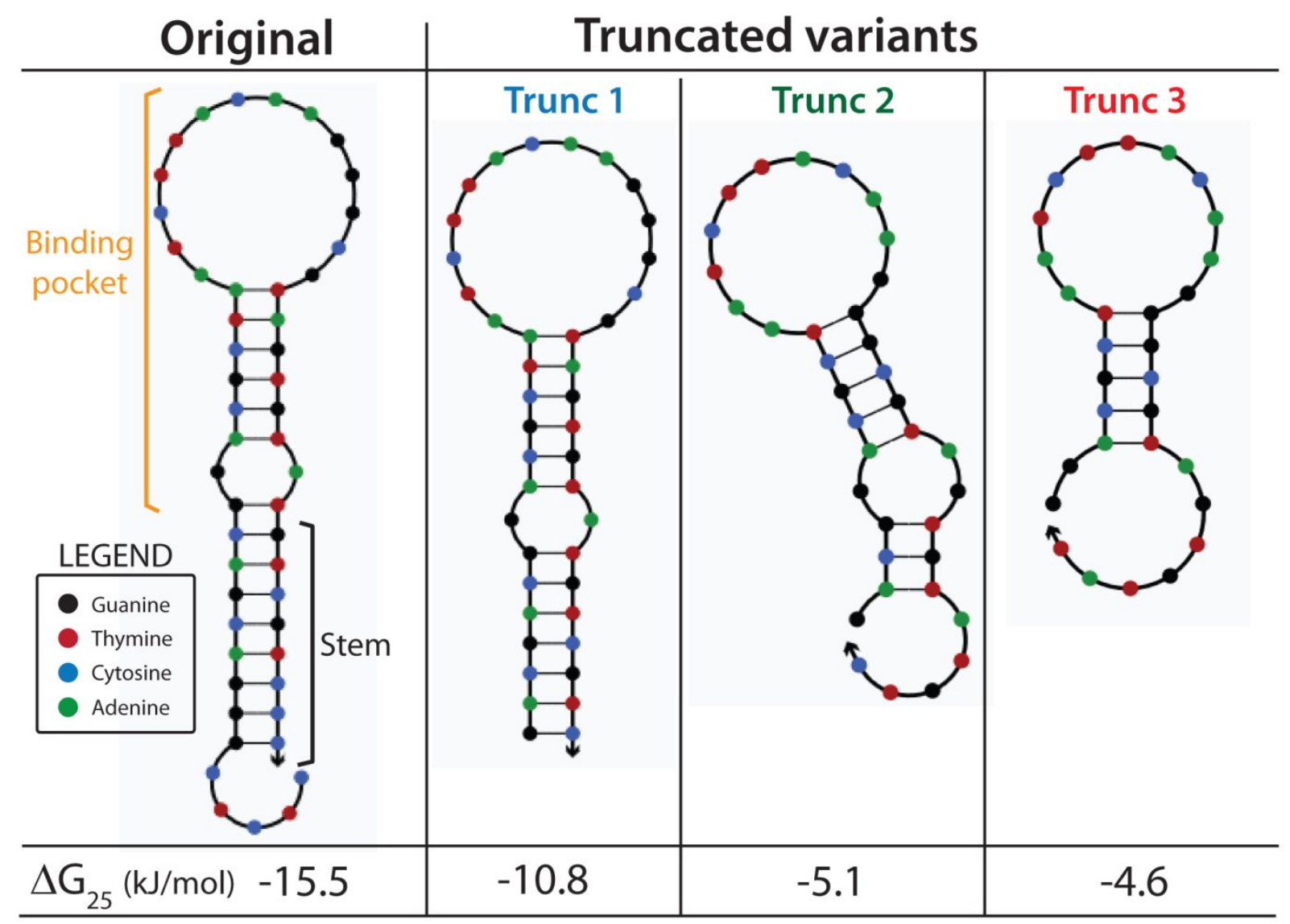

Figure S1. In an effort to create an optimally destabilized variant (to thus introduce bindinginduced folding) we produced and characterized several truncated sequences of the parent aptamer. Shown are the sequences and the NUPACK ${ }^{1}$ predicted folding thermodynamics and lowest-energy structure of each. 


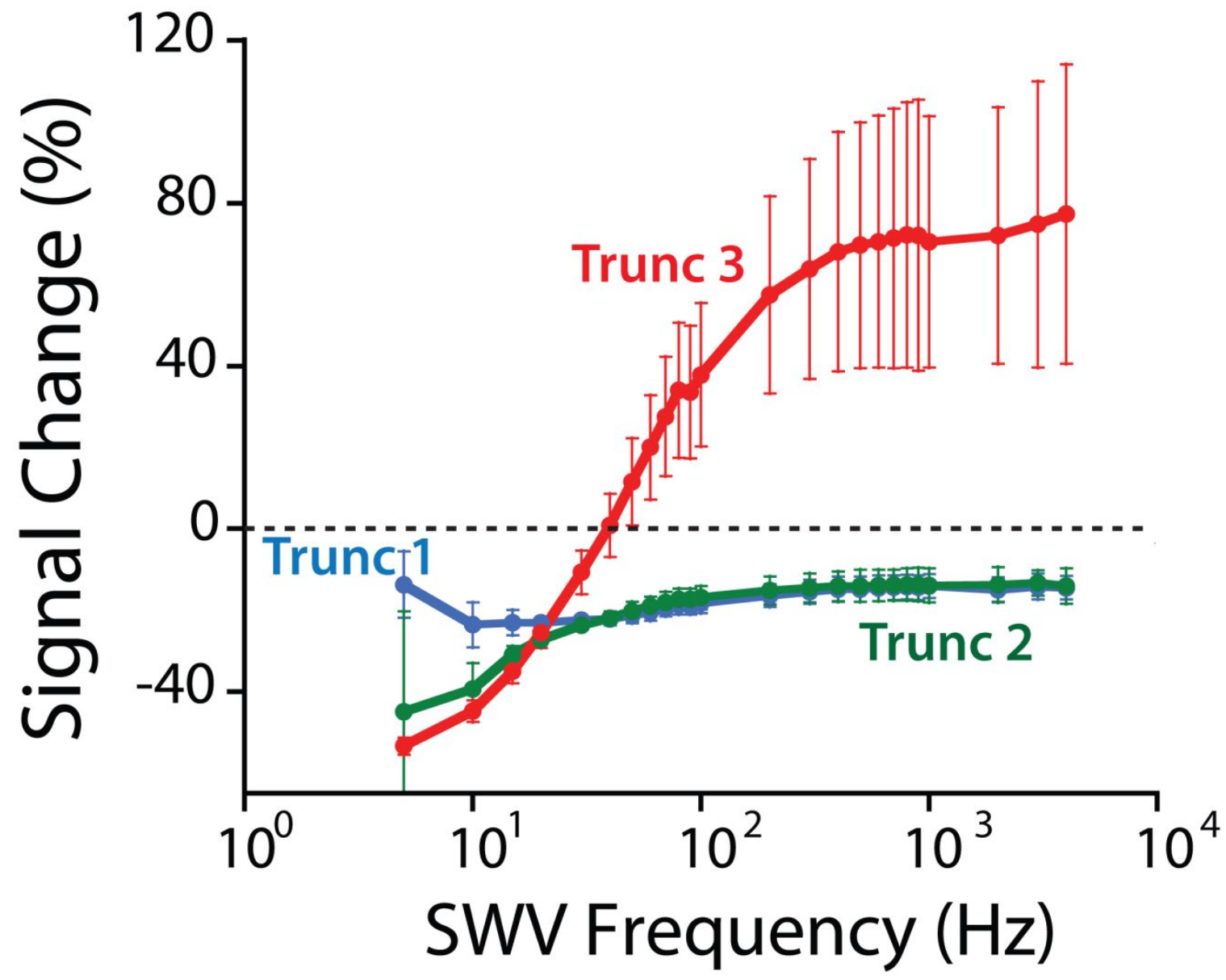

Figure S2. Sensors fabricated using all three truncates respond to phenylalanine. Here we have tested them over a range of square-wave frequencies in the absence and in presence of saturating $(100 \mu \mathrm{M})$ target and a fixed concentration $(100 \mu \mathrm{M})$ of the rhodium-based inorganic receptor. Shown is the relative gain (relative signal change between zero and saturating target) for all three sensors. 

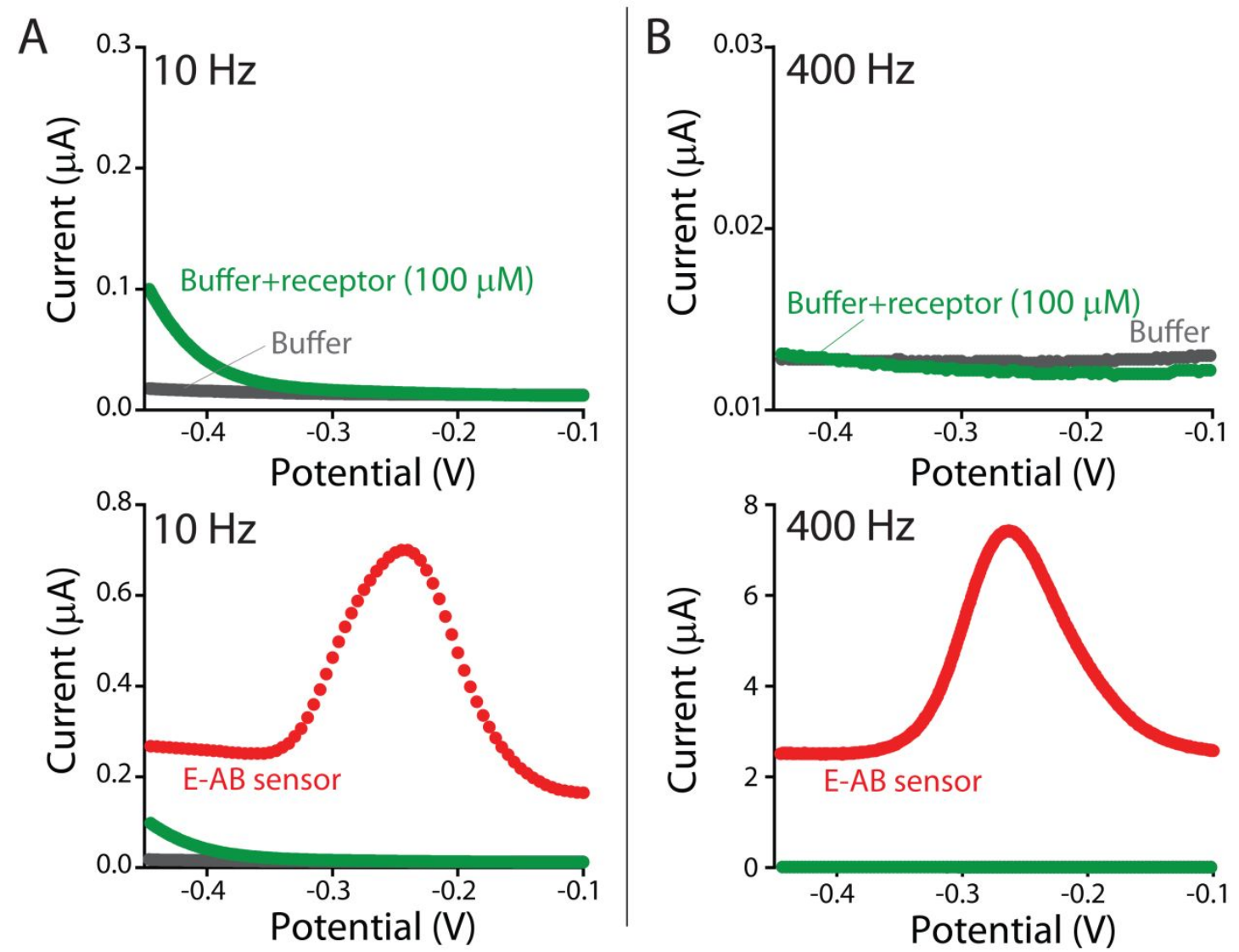

Figure S3. The rhodium receptor does not itself produce any measurable electrochemical signal over the potential window we have employed. (Top row) To demonstrate this we first interrogated electrodes modified only with 6-mercaptohexanol (i.e., no aptamer) in the presence and absence of the receptor at $100 \mu \mathrm{M}$. (Bottom row) By overlaying these same voltammograms with voltammograms collected from sensors with the aptamer under the same experimental conditions (i.e., in the presence of the receptor) illustrates the extent to which the small signal seen for the receptor at the far end of our potential window does not significantly alter E-AB signaling. 

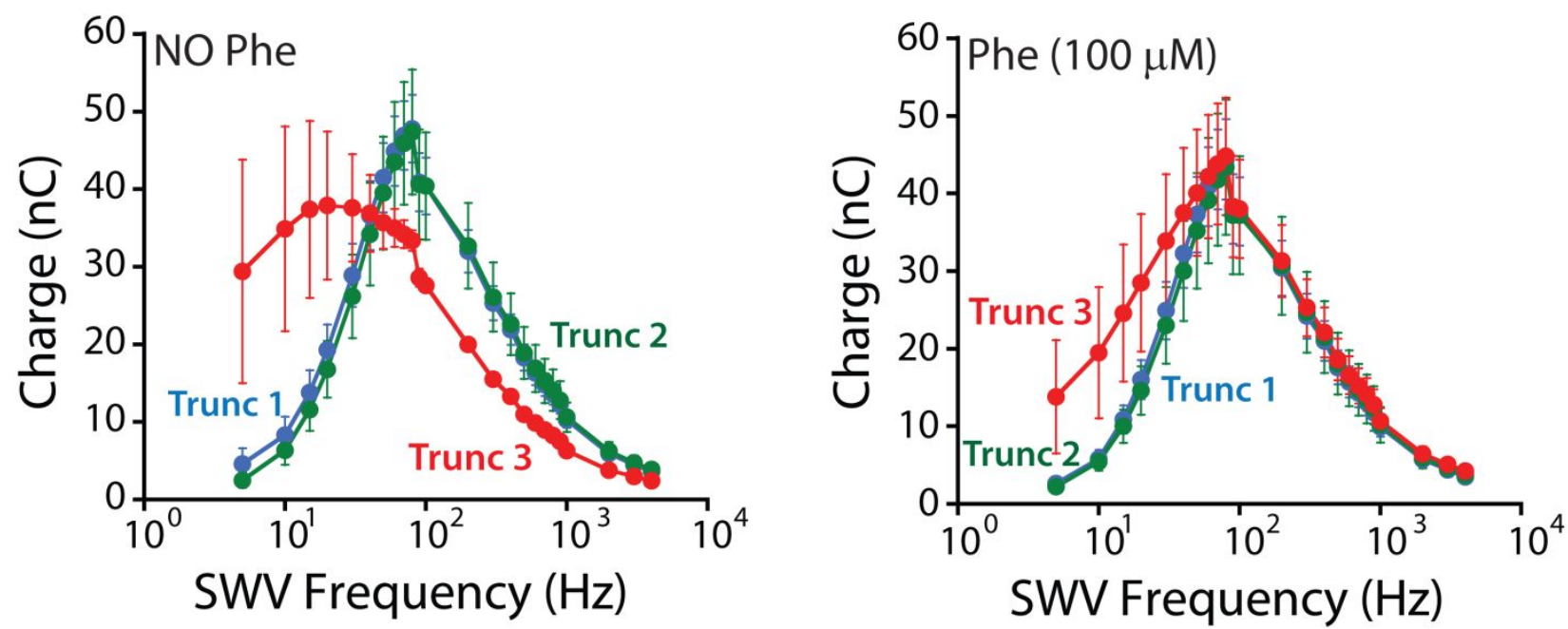

Figure S4. Using the ratio of the measured peak current to SWV frequency as a function of frequency we can estimate the apparent electron transfer rate of the methylene blue reporter for sensors employing each of our three aptamer variants. ${ }^{3}$ (Left) In the absence of the target the maximum transfer rate for Trunc 3 is $\sim 30 \mathrm{~Hz}$ and those of Trunc1 and Trunc2 are $\sim 100 \mathrm{~Hz}$. (Right) In contrast, all three variants exhibit the same electron transfer rate upon target binding, presumably because all three adopt the same bound-state conformation. 

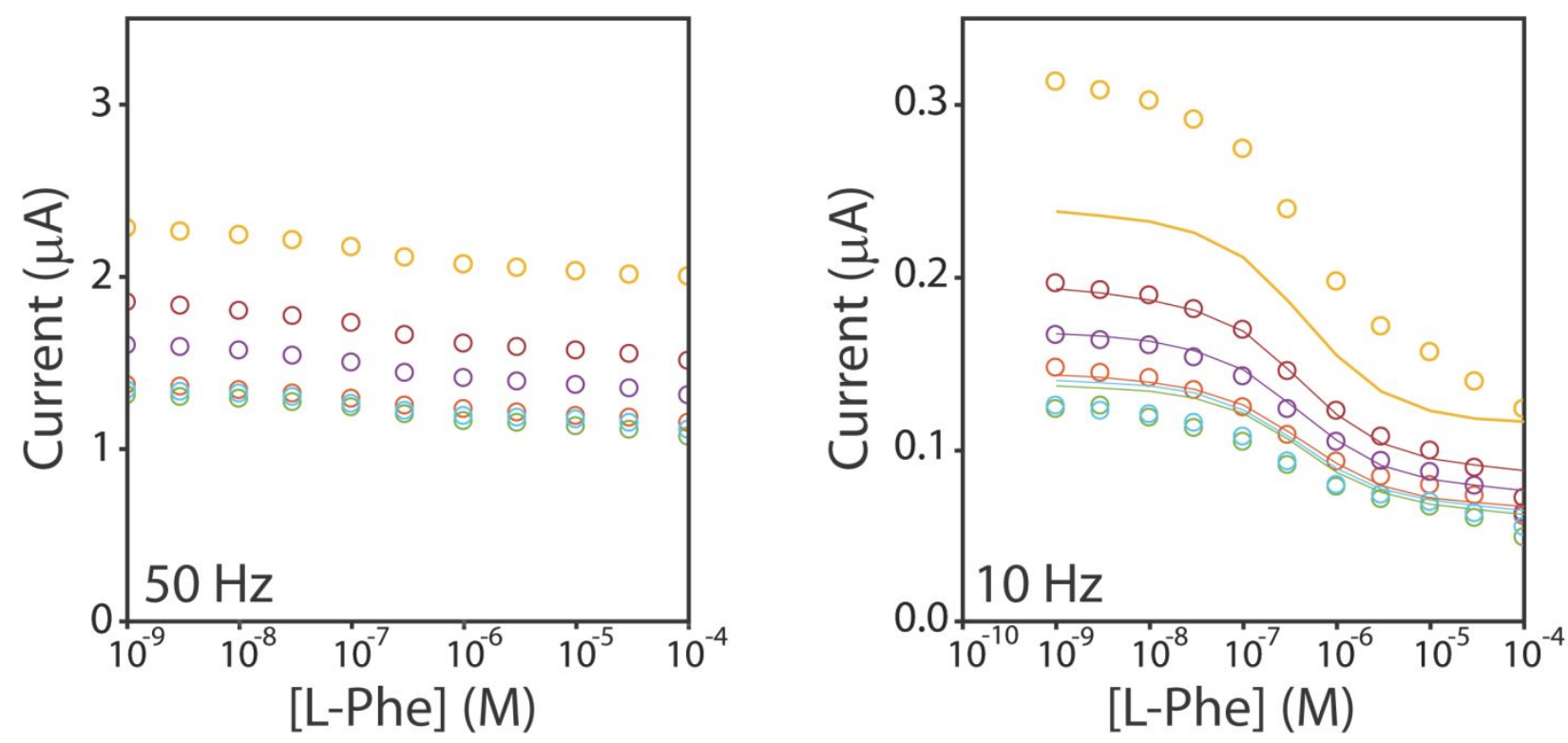

Figure S5. We used a global fit ${ }^{2}$ to define the parameters $K_{D}, \alpha$, and $\gamma$ for these phenylalaninedetecting sensors. Specifically, we fit the voltammetric signals of a training set of six sensors collected both at a non-responsive (or at least minimally responsive) frequency $(50 \mathrm{~Hz})$ and at a responsive frequency $(10 \mathrm{~Hz})$ to Eq. 1 by minimizing the least-square errors (Nelder-Mead simplex algorithm). We estimate the following values for the parameters: $K_{D}=0.56 \pm 0.12 \mu \mathrm{M}, \alpha=0.104 \pm$ $0.015, \gamma=-0.447 \pm 0.024$. On the right, the solid lines illustrate how well the globally fit parameters describe the voltammetric signal of each individual sensor.
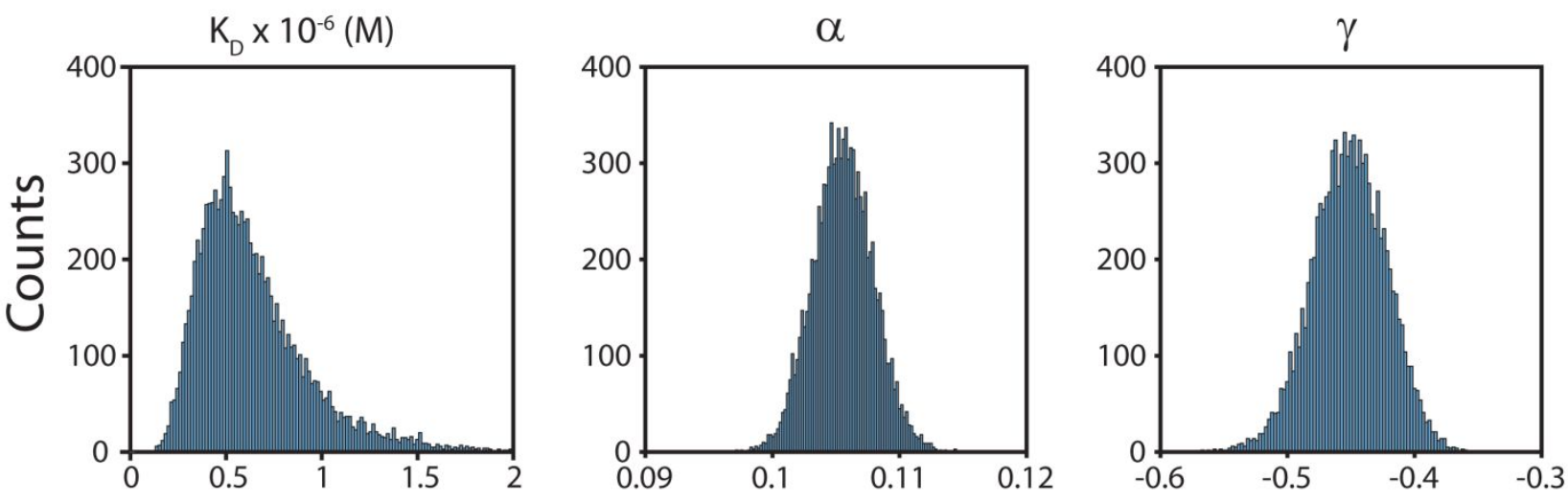

Figure S6. To estimate the uncertainties on the parameters for the calibration-free operation of the sensors (Figure S3) we performed Monte-Carlo analysis. Specifically, at each iteration we propagated the root-mean-square error into our experimental data and fit the new resulting dataset to Eq. 1 to extract a new set of parameters. We repeated this 10,000 times (enough iterations to ensure convergence) and then reported the uncertainties as the $95 \%$ confidence interval of the newly generated distribution of parameters. ${ }^{2}$ 

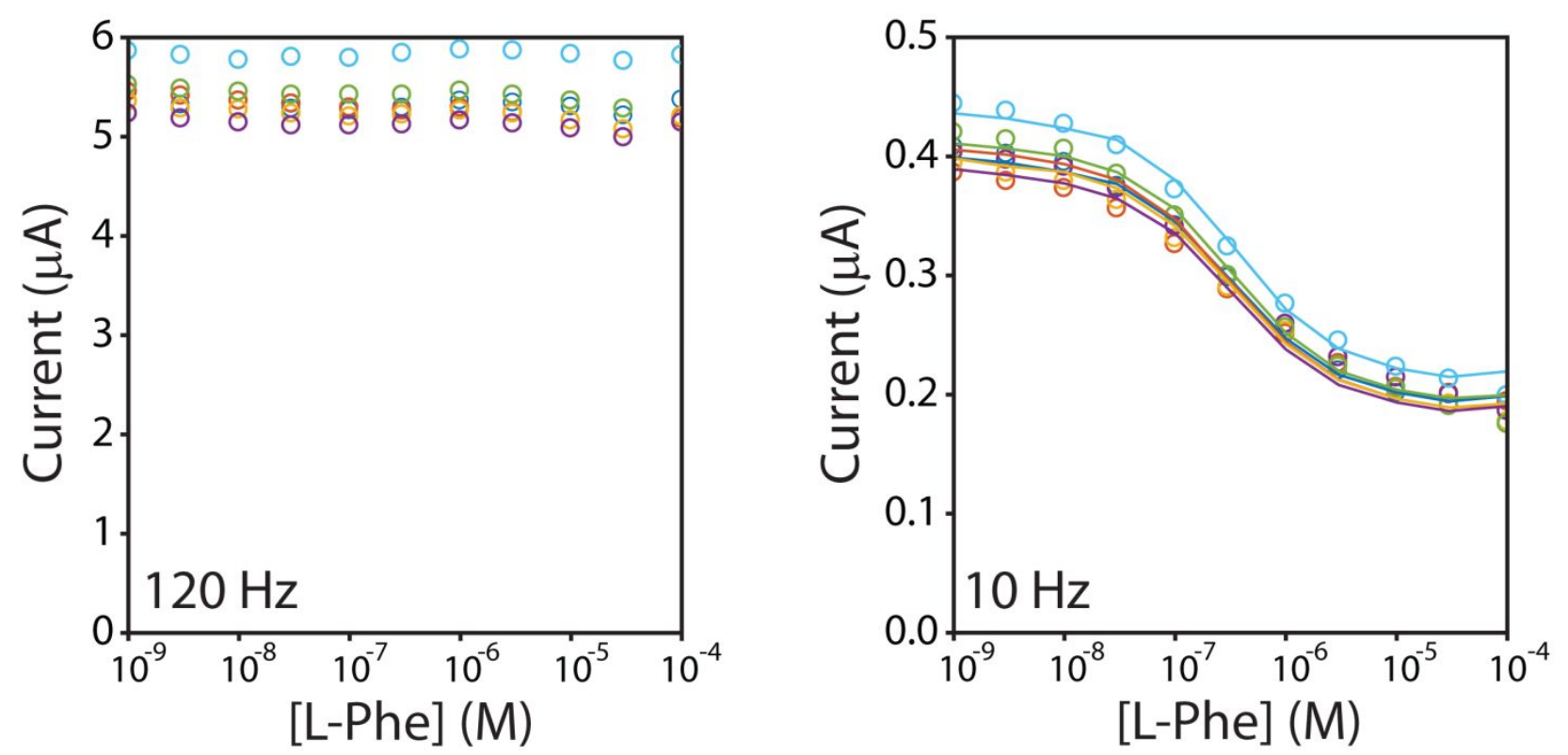

Figure S7. Determination of the parameters $K_{D}, \alpha$, and $\gamma$ for our phenylalanine-detecting sensor in diluted blood $(1: 1000){ }^{2}$ Our estimates for the relevant parameters are: $\mathrm{K}_{\mathrm{D}}=0.32 \pm 0.06 \mu \mathrm{M}, \alpha=$ $0.074 \pm 0.001, \gamma=-0.505 \pm 0.014$ The solid lines on the right illustrate how well the globally fit parameters describe the voltammetric signal of each individual sensor in the training set (right).
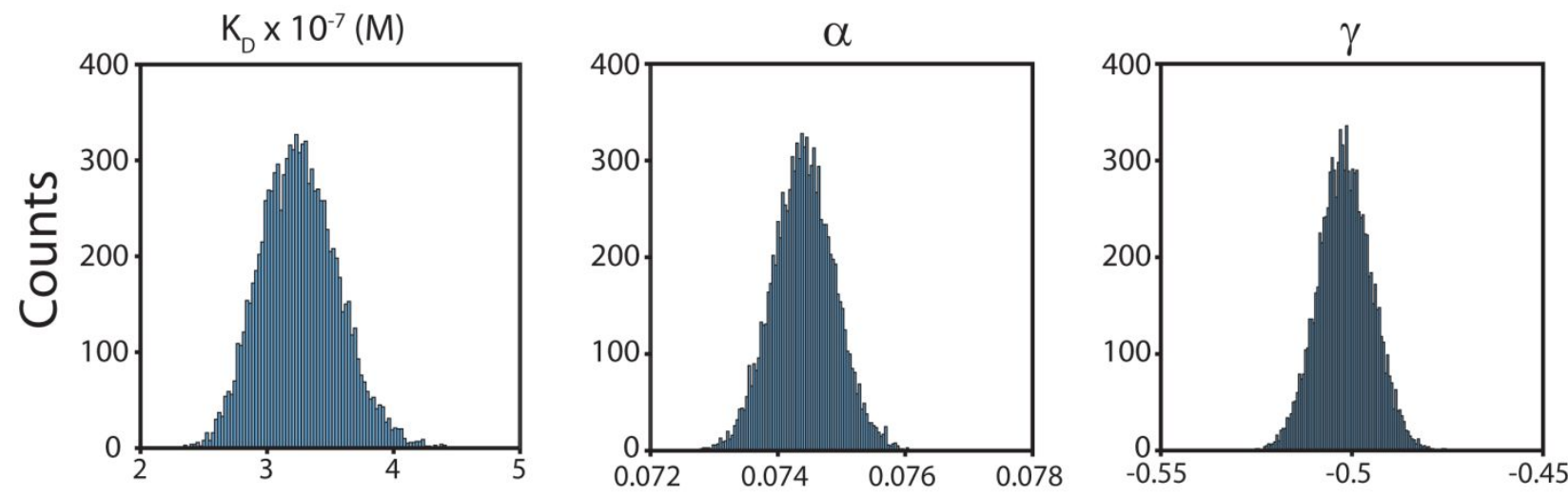

Figure S8. The variability in the calculated parameters $K_{D}$, $\alpha$, and $\gamma$, determined in figure $\mathrm{S} 5 .^{2}$ 


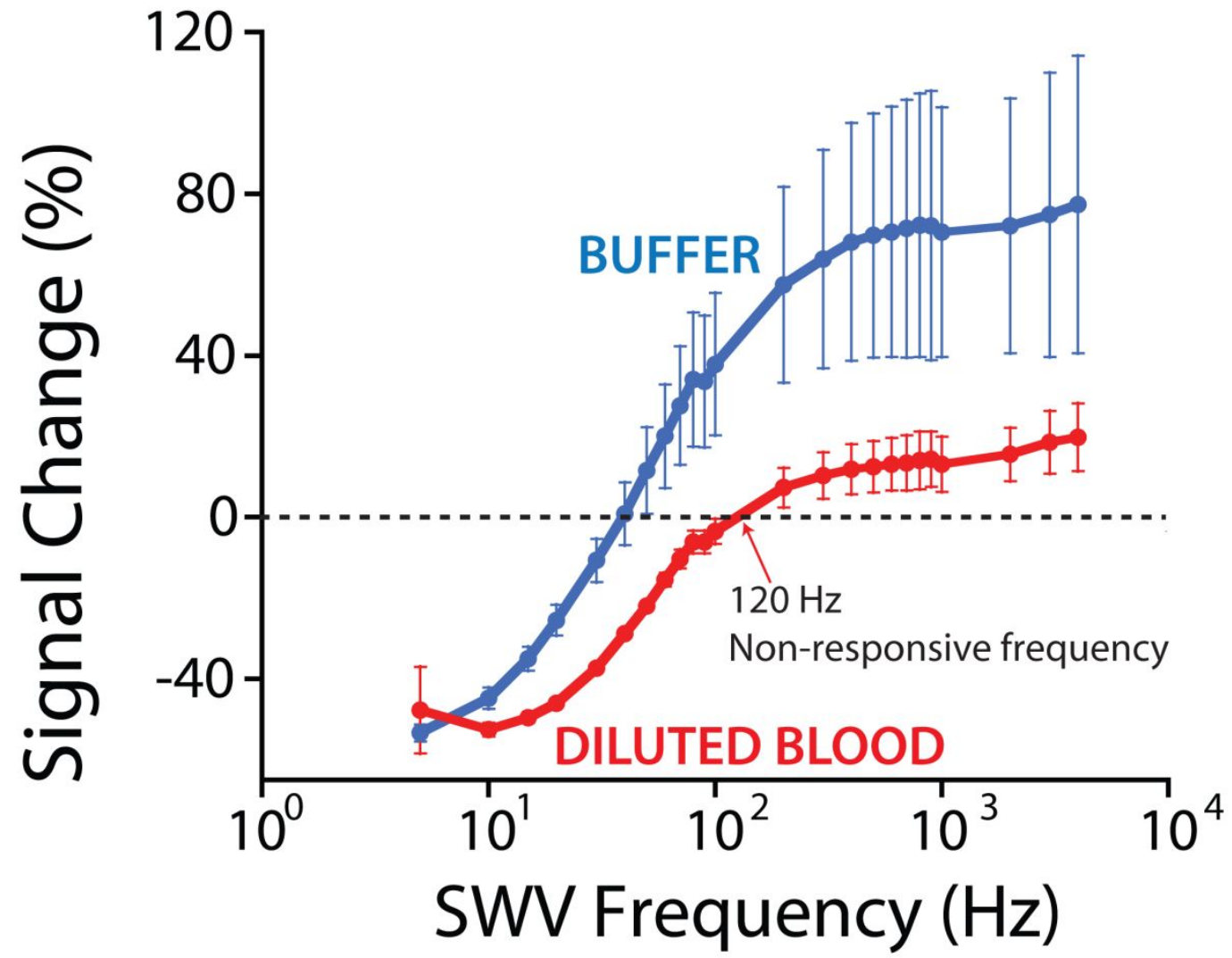

Figure S9. When we move our E-AB sensor to 1:100-diluted whole blood we observe a change in the non-responsive frequency, which we accounted for when we employed the dual-frequency calibration free approach. 


\section{References}

(1) Zadeh, J. N.; Steenberg, C. D.; Bois, J. S.; Wolfe, B. R.; Pierce, M. B.; Khan, A. R.; Dirks, R. M.; Pierce, N. A. NUPACK: Analysis and design of nucleic acid systems. J. Comput. Chem. 2011, $32,170-173$.

(2) Li, H.; Dauphin-Ducharme, P.; Ortega, G.; Plaxco, K. W. Calibration-Free Electrochemical Biosensors Supporting Accurate Molecular Measurements Directly in Undiluted Whole Blood. $J$. Am. Chem. Soc. 2017, 139, 11207-11213.

(3) Komorsky-Lovrić, Š.; Lovrić, M. Measurements of redox kinetics of adsorbed azobenzene by "a quasireversible maximum" in square-wave voltammetry. Electrochim. acta 1995, 40, 1781-1784. 